21st Particles and Nuclei International Conference (PANIC 2017)

International Journal of Modern Physics: Conference Series

Vol. 46 (2018) 1860038 (6 pages)

(C) The Author(s)

DOI: $10.1142 / \mathrm{S} 2010194518600388$

\title{
Results from the NOvA Experiment
}

\author{
Erica Smith (for the NOvA Collaboration) \\ Department of Physics, Indiana University, 727 East 3rd Street \\ Bloomington, Indiana 47405, USA \\ ess3@iu.edu
}

Published 3 May 2018

\begin{abstract}
The NOvA experiment is a long-baseline accelerator-based neutrino oscillation experiment. It uses the upgraded NuMI beam from Fermilab to measure electron-neutrino appearance and muon-neutrino disappearance between the Near Detector, located at Fermilab, and the Far Detector, located at Ash River, Minnesota. The NuMI beam has recently reached and surpassed the $700 \mathrm{~kW}$ power benchmark. NOvA's primary physics goals include precision measurements of oscillation parameters, such as $\theta_{23}$ and the atmospheric mass-squared splitting, along with probes of the mass hierarchy and of the CP violating phase. This talk will present the latest NOvA results, based on a neutrino beam exposure equivalent to $6.05 \times 10^{20}$ protons-on-target.
\end{abstract}

Keywords: NOvA; neutrinos; neutrino oscillations.

\section{Overview}

The NOvA experiment ${ }^{1}$, or the NuMI Off-Axis $\nu_{e}$ Appearance experiment, is a longbaseline neutrino oscillation experiment that uses the NuMI beam at Fermilab ${ }^{2,3}$. NOvA is composed of two functionally identical detectors located $14.6 \mathrm{mrad}$ off beam axis. The 300 ton Near Detector is located $1 \mathrm{~km}$ from the beam target 100 $\mathrm{m}$ underground at Fermilab, and the 14 kton Far Detector is located $810 \mathrm{~km}$ away at Ash River, MN, on the surface, with a $3 \mathrm{~m}$ overburden. The off-axis position maximizes the oscillation probability for the electron neutrino appearance channel and results in a muon neutrino energy spectrum that peaks at $2 \mathrm{GeV}$, giving us a distance-to-energy ratio located at the first oscillation maximum for oscillations driven by $\Delta m_{32}^{2}=2.5 \times 10^{-3} \mathrm{eV}^{2}$.

This talk describes neutrino oscillation analyses with the first $6.05 \times 10^{20}$ protonson-target (POT) of full-detector equivalent exposure in neutrino mode, as described in Refs. 4, 7, 10. NOvA has since added $3 \times 10^{20}$ POT in anti-neutrino mode.

This is an Open Access article published by World Scientific Publishing Company. It is distributed under the terms of the Creative Commons Attribution 4.0 (CC-BY) License. Further distribution of this work is permitted, provided the original work is properly cited. 


\section{Physics Goals}

The primary physics goal of NOvA is to observe oscillations in the muon neutrino NuMI beam by comparing neutrino spectra between the Near and Far Detectors. Making these comparisons allows us to measure the neutrino oscillation parameters. NOvA can make precise measurements of the mixing angle $\theta_{23}$ and its octant, the neutrino mass hierarchy, and the CP-violating phase in the lepton sector $\delta_{C P}$ by measuring $\nu_{\mu} \rightarrow \nu_{e}$ and $\bar{\nu}_{\mu} \rightarrow \bar{\nu}_{e}$ transitions. The $\nu_{\mu} / \bar{\nu}_{\mu}$ disappearance analysis allows for measurement of $\Delta m_{32}^{2}$ and $\sin ^{2}\left(\theta_{23}\right)$. The $\nu_{e} / \bar{\nu}_{e}$ appearance analysis allows for measurement of $\theta_{13}, \theta_{23}, \delta_{C P}$, and the mass hierarchy. The neutral current (NC) analysis looks for suppression of NC events which may be an indicator of oscillations involving a sterile neutrino, parameterized by $\Delta m_{41}^{2}, \theta_{34}$, and $\theta_{24}$. Many other non-oscillation studies are done with the NOvA detectors, including crosssection studies, exotic phenomena, and non-beam physics studies; these studies are not covered in this talk.

\section{Detectors}

The functionally identical detectors are segmented, tracking calorimeters. The detectors are comprised of $4-\mathrm{cm}$ by $6-\mathrm{cm}$ cells made of extruded PVC and filled with organic scintillator, which constitutes $62 \%$ of their fiducial mass. Low $Z$ materials are used, resulting in a radiation length of about $40 \mathrm{~cm}$; this allows for 6 samples per radiation length longitudinally and 3 samples per Moliere radius transversely, making showers from the decay of electrons and neutral pions distinguishable. Wavelength shifting fibers collect light in the cells, which are read out by avalanche photodiodes. Cells are arranged in planes, which alternate horizontal and vertical orientations, enabling three-dimensional event reconstruction.

The NuMI beam from Fermilab, currently operating at its design goal of $700 \mathrm{~kW}$, is created using $120 \mathrm{GeV}$ protons from the Main Injector which create charged kaons and pions, which decay, producing anti-muons and muon neutrinos. The charged particles are focused with magnetic horns, allowing the selection of neutrinos or anti-neutrinos. The flavor composition of the beam at the Near Detector, estimated from simulations and assuming no oscillations, is $97.5 \% \nu_{\mu}, 1.8 \% \bar{\nu}_{\mu}$, and $0.7 \% \nu_{e}$ $+\bar{\nu}_{e}$.

\section{Muon Neutrino Disappearance}

The composition and energy spectra of the NuMI beam are measured first at the Near Detector, before oscillations. These measurements are extrapolated to form a prediction of the spectrum at the Far Detector. Far Detector data is then compared to the predictions to estimate the oscillation parameters.

Different neutrino flavors can be distinguished in the NOvA detectors, as seen in Fig. 1, by the topology of the outgoing particles. Containment and event topology requirements are used to remove backgrounds from cosmic rays and NC events. A 


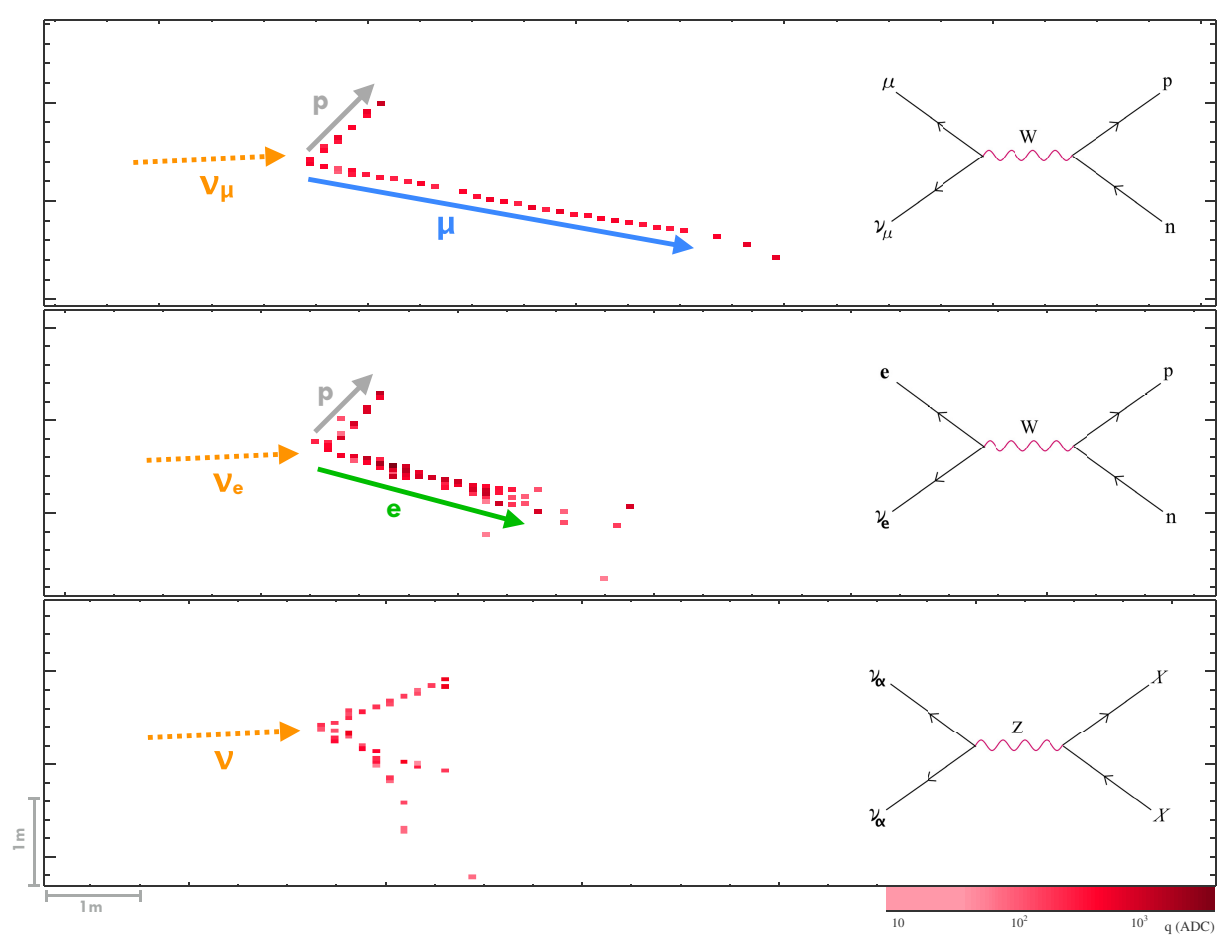

Fig. 1. Sample event topologies in NOvA. Three different neutrino interactions are shown: top is a selected $\nu_{\mu}$ charged current event; middle is a selected $\nu_{e}$ charged current event; bottom is a NC event. Each pixel corresponds to one cell in the detector. The characteristic feature of the top interaction is the long, low $d E / d x$ track that corresponds to a minimally ionizing muon. The signature of the middle interaction is a shorter, broader electromagnetic shower. The bottom interaction is a NC event; the only signature of these events is the hadronic component. In this event, a neutral pion is produced which decays into two photons. The distinguishing feature between this interaction and a $\nu_{e}$ interaction is the gap between the interaction vertex and the shower.

traditional $k$-Nearest Neighbor $(k \mathrm{NN})$ is used to select $\nu_{\mu} \mathrm{CC}$ events; this selection is $62 \%$ efficient. The energy of the selected muon tracks is calculated using a length-to-energy conversion. The hadronic energy is estimated from the sum of the calorimetric energy of the remaining non-muon hits. The neutrino energy is the sum of these two values; the energy resolution at the beam peak is estimated to be $7 \%$.

With this method, we select $78 \nu_{\mu}$ candidate events, shown in Fig. 2a. Without oscillations, we predict $473 \pm 30$ events. Of the selected 78 candidate events, there are an estimated 2.7 cosmic, $3.4 \mathrm{NC}, 0.2 \nu_{e}$, and $0.3 \nu_{\tau}$ events, indicated by the blue line in Fig. 2a. Oscillations are fit to the spectra, producing a best fit of $\Delta m_{32}^{2}=(+2.67 \pm 0.11) \times 10^{-3} \mathrm{eV}^{2}$ and $\sin ^{2} \theta_{23}$ at degenerate values $0.404_{-0.022}^{+0.030}$ and $0.624_{-0.030}^{+0.022}(68 \%$ C.L.) in the normal hierarchy; for the inverted hierarchy, $\Delta m_{32}^{2}=(-2.72 \pm 0.11) \times 10^{-3} \mathrm{eV}^{2}$ and $\sin ^{2} \theta_{23}$ at degenerate values $0.398_{-0.022}^{+0.030}$ and $0.618_{-0.030}^{+0.022}$. The resulting allowed regions for $\Delta m_{32}^{2}$ and $\sin ^{2} \theta_{23}$ are shown in 


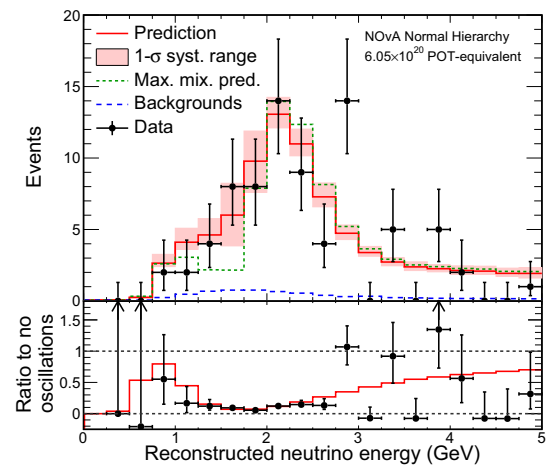

(a)

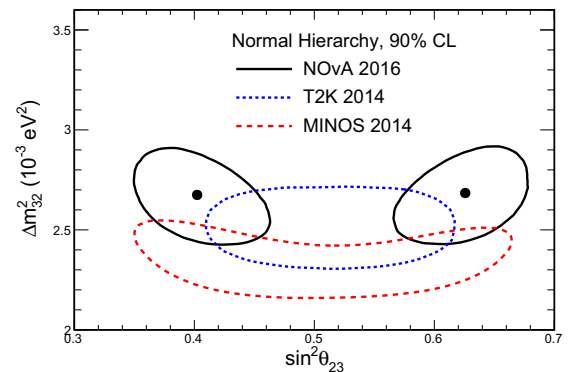

(b)

Fig. 2. (a) The top panel shows the neutrino energy spectrum including data, best fit prediction, systematics band about stats-only best fit (not shown), prediction at maximal mixing, and backgrounds. The bottom panel shows the spectrum ratio of data over unoscillated prediction and best fit prediction over unoscillated prediction. All spectra have the predicted backgrounds subtracted out before division. (b) Feldman-Cousins corrected $2 \mathrm{D}$ confidence intervals, with recent $\mathrm{T}_{2} \mathrm{~K}^{5}$ and MINOS $^{6}$ results.

Fig. 2b. Maximal mixing is disfavored at $2.6 \sigma$; for a full discussion of this analysis please see Ref. 4 .

\section{Neutral Current Disappearance}

The primary goals of the $\mathrm{NC}$ analysis ${ }^{7}$ is to search for evidence for sterile neutrinos and constrain mixing between the three known active neutrinos and one or more light sterile neutrinos. NOvA is primarily sensitive to sterile neutrino mixing via disappearance of NC events. The NC spectrum is insensitive to standard threeflavor mixing, so observation of an energy-dependent depletion of the neutrino flux at the Far Detector would be a signature of active-sterile mixing.

The search for active-sterile neutrino oscillations compares the predicted FD rate with the observed $\mathrm{NC}$ events; the $\mathrm{FD}$ rate is predicted from the calorimetric energy for NC-selected events. This analysis predicts 83.5 events under the 3 -flavor assumption and observes $95 \mathrm{NC}$ events. This count is $1.03 \sigma$ above and consistent with the 3 -flavor; that is, no sterile mixing. The allowed regions for the sterile mixing angles $\theta_{24}$ and $\theta_{34}$ are shown in Fig. 3a, and relevant mixing matrix elements $\left|U_{\mu 4}\right|^{2}$ and $\left|U_{\tau 4}\right|^{2}$ in Fig. $3 \mathrm{~b}$.

\section{Electron Neutrino Appearance}

Electron neutrino events are selected using the convolution visual network (CVN), which is based on ideas from computer vision and deep learning ${ }^{11}$. Using CVN improves the sensitivity of the result by $30 \%$ over traditional algorithms; the resulting $\nu_{e}$ selection has an efficiency of $73.5 \%$ and $75.5 \%$ purity. It also rejects $97.6 \%$ of the NC backgrounds and $99.0 \%$ of the $\nu_{\mu}$ charged current beam backgrounds. 


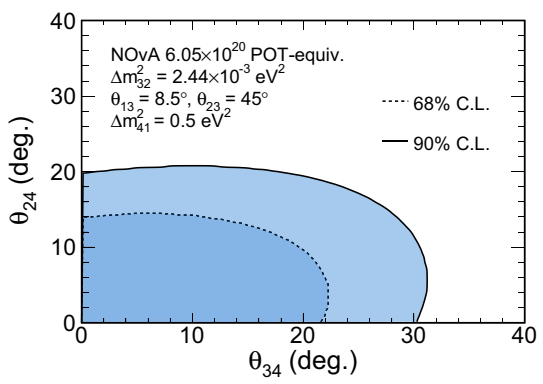

(a)

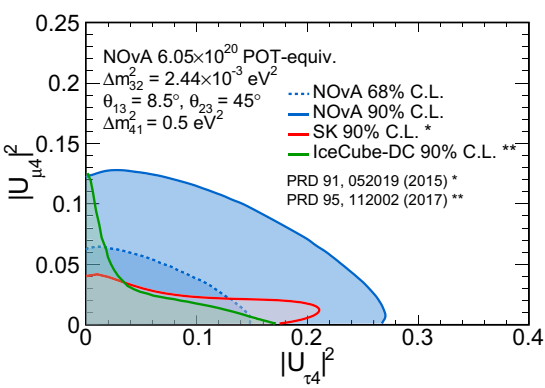

(b)

Fig. 3. (a) Feldman-Cousins corrected 2D confidence intervals (shaded) at $68 \%$ and $90 \%$ C.L. for the sterile mixing angles $\theta_{24}$ and $\theta_{34}$. We set limits of $\theta_{24}<20.8^{\circ}$ and $\theta_{34}<31.2^{\circ}$ at the $90 \%$ C.L. (b) Feldman-Cousins corrected 2D confidence intervals (shaded) at $68 \%$ and $90 \%$ C.L. in terms of $\left|U_{\mu 4}\right|^{2}$ and $\left|U_{\tau 4}\right|^{2}$ where we assume $\cos ^{2} \theta_{14}=1$ in both cases. We set limits of $\left|U_{\mu 4}\right|^{2}$ $<0.126$ and $\left|U_{\tau 4}\right|^{2}<0.268$ at the $90 \%$ C.L. Includes recent results from Super-Kamiokande ${ }^{8}$ and IceCube-DeepCore ${ }^{9}$.

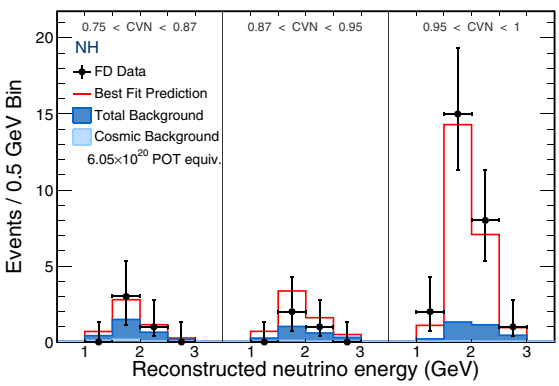

(a)

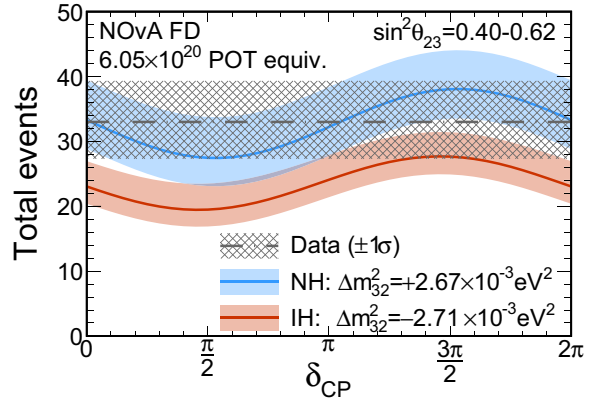

(b)

Fig. 4. (a) Reconstructed energy of selected Far Detector events in three bins of the CVN classifier variable; data shown in black, total expected background in blue, and predicted spectrum at best fit point in normal hierarchy in red. (b) Total number of events observed in the Far Detector (gray) and $1 \sigma$ uncertainty, overlaid with the expectation as a function of $\delta_{C P}$ for normal hierarchy (blue) and inverted hierarchy (red), with fixed $0.404<\sin ^{2} \theta_{23}<0.623$.

Near Detector $e$-like data is used to predict the background in the Far Detector, including electron neutrinos from the beam itself, $\nu_{\mu} \mathrm{CC}$ interactions, and NC interactions. With this selection, $33 \nu_{e}$ candidates are observed, which is an $8 \sigma$ electron neutrino appearance signal over a background of 8.2 events. For a more detailed description of the electron neutrino analysis see Ref. 10.

The spectrum is fit for hierarchy, $\delta_{C P}$, and $\sin ^{2} \theta_{23}$. Results from the $\nu_{\mu}$ disappearance analysis constrains $\Delta m^{2}$ and $\sin ^{2} \theta_{23}$, while $\theta_{13}$ is constrained with results from reactor experiments ${ }^{12}$. The global best fit point is in the normal hierarchy, with degenerate best fit points at $\delta_{C P}=1.48 \pi$ and $\sin ^{2} \theta_{23}=0.404$, and $\delta_{C P}=0.74 \pi$ with $\sin ^{2} \theta_{23}=0.623$. The inverted hierarchy is rejected at greater than $90 \%$ C.L. for $\delta_{C P}$ near $\pi / 2$ with $\theta_{23}$ in the lower octant. 


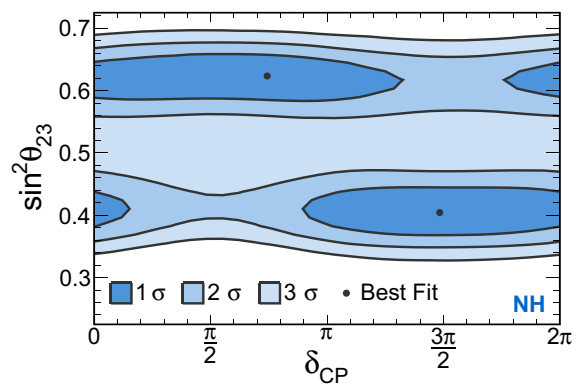

(a)

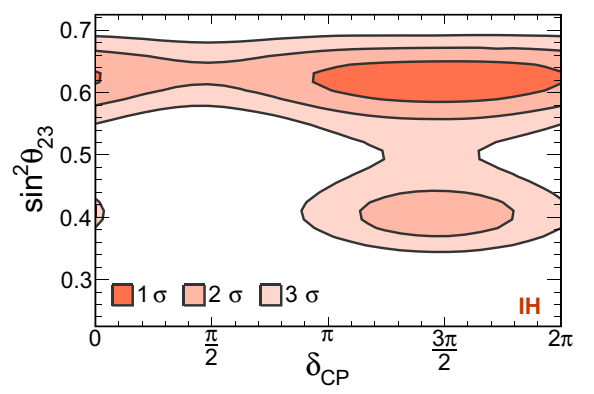

(b)

Fig. 5. Allowed regions of $\delta_{c p}$ vs. $\sin ^{2} \theta_{23}$ parameter space for the (a) normal hierarchy and (b) inverted hierarchy. Color intensity indicates the confidence level.

\section{Summary}

Using $6.05 \times 10^{20}$ POT, NOvA indicates a non-maximal best fit for $\theta_{23}$ and disfavors maximal mixing at $2.6 \sigma$. NOvA sees a slight preference for the normal hierarchy and rejects the inverted hierarchy for the lower octant and $\delta_{C P} \sim \pi / 2$. Running in antineutrino mode will resolve the degeneracies caused by the $\theta_{23}$ octant; data-taking in anti-neutrino mode began in February 2017.

\section{References}

1. NOvA Collaboration (D. S. Ayres et al.), The NOvA Technical Design Report, FERMILAB-DESIGN-2007-01.

2. K. Anderson et al., The NuMI Facility Technical Design Report, FERMILABDESIGN-1998-01.

3. MINOS Collaboration (P. Adamson et al.), Nucl. Instrum. Meth. A 806, 279 (2016).

4. NOvA Collaboration (P. Adamson et al.), Phys. Rev. Lett.118, 151802 (2017).

5. K. Abe et al., Phys. Rev. D 91, 072010 (2015).

6. P. Adamson et al., Phys. Rev. Lett. 112, 191801 (2014).

7. NOvA Collaboration (P. Adamson et al.), Phys. Rev. D 96, 072006 (2017).

8. Super-Kamiokande Collaboration (K. Abe et al.), Phys. Rev. D 91, 052019 (2015).

9. IceCube Collaboration (M. G. Aartsen et al.), Phys. Rev. D 95, 112002 (2017).

10. NOvA Collaboration (P. Adamson et al.), Phys. Rev. Lett. 118, 231801 (2017).

11. A. Aurisano et al., JINST 11, P09001 (2016).

12. Particle Data Group (C. Patrignani et al.), Chin. Phys. C, 40, 10, 100001 (2016). 